\title{
Painful Goiter: A Rare Presentation of Graves' Disease
}

\author{
Lakshmi Kannan a, , Young-Nam Kim ${ }^{\mathrm{a}}$
}

\begin{abstract}
Here we report a rare case of Graves' disease presenting as subacute thyroiditis. In the literature, only two reports are available of such a presentation. Various authors have reported in total 33 patients with subacute thyroiditis who were subsequently, between 1 and 18 months later, diagnosed with Graves' disease. However, only two patients with both subacute thyroiditis and Graves' disease simultaneously have been reported. Subacute thyroiditis is an inflammatory disorder while Graves' disease has an autoimmune basis. Although the association of the two could be incidental, it could be postulated that thyroiditis exposes autoantigens, enhancing antibody formation in susceptible patients and thereby precipitating or unmasking Graves' disease. Hence, it is important to remember that though rare, Graves' disease can present with painful goiter due to concurrent thyroiditis, or rather is unmasked by the inflammation.
\end{abstract}

Keywords: Graves'; Painful goiter; Thyroiditis

\section{Introduction}

Graves' disease is a well-known autoimmune disorder of the thyroid gland that usually presents with a painless goiter. Subacute thyroiditis (SAT) is a distinct inflammatory condition of the thyroid that is associated with a tender goiter. It is the most common cause of thyroid pain. The pathophysiology of these two conditions is distinct. Meticulous clinical history, physical examination and radio-active Iodine uptake

Manuscript accepted for publication December 9, 2013

${ }^{a}$ Einstein Medical Center, Philadelphia, PA, USA

${ }^{\mathrm{b}}$ Corresponding author: Lakshmi Kannan, Einstein Medical Center, Department of Internal Medicine, Klein Building, 5501 Old York Road, Philadelphia, PA 19141, USA. Email: Kannanla@einstein.edu

doi: http://dx.doi.org/10.14740/jmc1626w
(RAIU) scans are commonly used to distinguish the two conditions. Management varies significantly for transient thyroid inflammation in SAT as compared to autoimmune hyperthyroidism from Graves' disease. Though very rare, these two conditions have been reported to occur simultaneously. Review of the literature shows multiple pathological explanations have been postulated for the co-occurrence of Graves' disease and SAT in a given patient.

Through this report of such a rare occurrence, we would like to describe the importance of clinical findings in thyroid pathologies, the role of RAIU and possible immunologic mechanisms for the association of SAT and Graves' disease.

\section{Case Report}

A 20-year-old female presented with acute onset, 2-day duration neck pain and swelling during her fourth postpartum month. The pain was radiating to her ears, associated with fever and odynophagia. She reported palpitations, excessive sweating and tremors that started at the same time as the pain. She had weight loss of about 10 pounds in the preced-

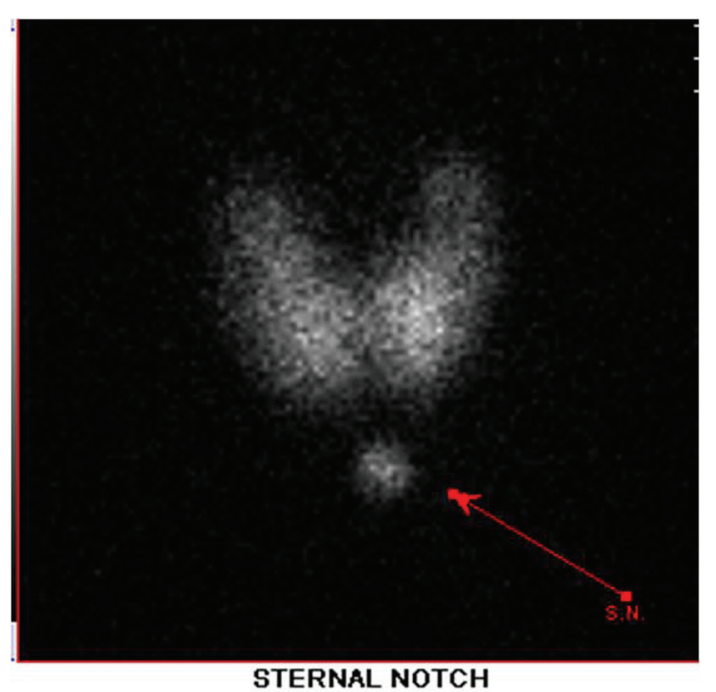

Figure 1. Radioactive iodine uptake showing diffusely increased uptake. 
ing few weeks.

On physical examination, she was an anxious looking female with warm skin. Fine tremors were noticed in her hands. An exquisitely tender goiter with diffuse enlargement of both lobes of the thyroid was present. Auscultation of the neck revealed bilateral bruit.

Labs showed TSH $<0.01 \mathrm{mcIU} / \mathrm{mL}$, T4 $14.7 \mathrm{mcg} / \mathrm{dL}$, free T4 $4.89 \mathrm{ng} / \mathrm{dL}$, total T3 $407.21 \mathrm{ng} / \mathrm{dL}$ and ESR 36.

The patient was started on treatment for subacute thyroiditis (SAT) with NSAIDs and propranolol. Due to severe persistent pain, she also received oral steroids. Radioactive iodine uptake (RAIU) study was performed the following day. The findings of diffusely enhanced uptake of $81 \%$ in the thyroid confirmed Graves' disease (Fig. 1).

\section{Discussion}

Here we report a rare case of Graves' disease presenting in the postpartum period as SAT. In the literature, only two reports are available of the simultaneous presentation of SAT and Graves' disease but none in postpartum patients $[1,2]$.

The causes of painful goiter in general are very few. The major ones are acute purulent thyroiditis, SAT, hemorrhage into a cyst or tumor. SAT is an inflammatory disorder of the thyroid gland attributed to viral infection. It is the most common cause of thyroid pain though the incidence of SAT itself is low in the population; the annual incidence is reported to be around 4 to 5 cases per 100,000 population. The disease most often affects patients in the 40 s to 50 s with a $4: 1$ female to male ratio. The most common presenting features include neck pain, low-grade fever and myalgias. The pain may radiate to the ears or be exacerbated by swallowing. Tender diffuse goiter on examination is the classic finding. SAT is a rare cause of postpartum thyrotoxicosis. Though based on her symptoms alone our patient fits into the diagnosis of SAT, physical examination revealed an unexpected finding of bilateral thyroid bruit. SAT is not associated with a bruit. Presence of bruit indicated hypervascularity and urged us to further investigate with RAIU scan to look for underlying autoimmune disease of the thyroid.

Rare patients with focal increases in uptake have been reported, but diffusely enhanced RAIU is not a feature of SAT. SAT is associated with a low RAIU [3, 4]. There have been individual reports of a moderately high RAIU in SAT. Sumie et al report a patient with SAT and RAIU of $17 \%$ that they thought was transitory due to high Trab levels [5]. In their paper, they also mention that few other authors have reported high RAIU in SAT patients. We do not think that this was the case with our patient who had a very high diffuse uptake associated with typical signs of Graves' disease. Graves' disease is an autoimmune thyroid disorder (AITD) leading to hyperstimulation of the gland by autoantibodies. It has a female preponderance with a threefold increased incidence in the postpartum period. Bruit is a characteristic feature of Graves' disease wherein thyroid hyperstimulation leads to enhanced blood flow. Thus our patient had a very unique presentation of two different etiologies of hyperthyroidism simultaneously during the postpartum period.

A thorough literature review revealed interesting reports of patients receiving a diagnosis of Graves' disease months after the occurrence of SAT. The association of SAT and Graves' is very rare. A review reported Graves' manifesting in SAT patients months after the remission of SAT and the RAIU at the time of SAT was low but high during the subsequent Graves' presentation. Various authors have reported in total 33 patients with SAT who were subsequently diagnosed with Graves' disease [6].

Given the differing pathogeneses of SAT and Graves' disease, it would be interesting to look into possible common pathways linking them. It is accepted widely that SAT is the result of follicular destruction by an inflammatory response to viral infections. The disease follows a triphasic course beginning with the thyrotoxic phase produced by the release of preformed thyroid hormones into the circulation. This is followed by the hypothyroid phase and over a period of months, the patient returns to euthyroid status [3]. It has been reported that in SAT, damages to the thyroid follicles lead to the immunologic activation of the thyroid by exposing autoantigens. Similar mechanisms have been postulated for the increased incidence of Graves' disease following thyroid injury from radiation. Recently Umut et al have reported the occurrence of SAT in Hashimoto's thyroiditis which is another disorder of the thyroid with autoimmune etiology. Graves' disease has also been reported in patients with Hashimoto's thyroiditis. One could postulate that in genetically susceptible individuals, exposure of autoantigens from the thyroid follicles during an episode of SAT facilitates an autoimmune response ultimately leading to overt Graves' disease. At least seven genes and their variants have been associated with AITD, including the histocompatibility complex, $H L A-D R$ gene locus and two other groups, immune regulatory genes (cytotoxic T lymphocyte-associated protein $4, C D 40$, protein tyrosine phosphatase-22 and CD25) and thyroid-specific genes (thyroglobulin and thyrotropin receptor genes).

Another mechanism reported in the role of environmental triggers in Graves' is the alterations in HLA expression. Thyroid cells of Graves' patients but not normal individuals express HLA molecules. Experimental models of mice have demonstrated that viral infections of the thyroid activate HLA MHC expression on the thyroid cells leading to activation of immune response. Direct viral infection of epithelial cells or cytokine activation by infection and subsequent activation has been proposed and confirmed in experimental models [7]. The following sequence has been proposed: local viral infection causes interferon production, or other local environmental factors which would induce DR expression, presentation of autoantigens and subsequent autoim- 
mune T-cell induction. These $\mathrm{T}$ cells would activate effector $\mathrm{B}$ and T cells. Whether this sequence leads to overt Graves' would depend on the genetic constitution of the individual.

While thyroid antibodies have been reported in patients with typical SAT, they have been linked to transitory autoimmune phenomena. One paper though extrapolates this mechanism to the rare development of Graves' in patients with SAT [6]. Abnormalities of the suppressor T-cell pathway have also been reported to coexist with autoimmunity and necessary to induce autoimmune disease in mice [9].

Another mechanism proposed was the inherent predisposition to both SAT and Grave's. In their report, on the simultaneous presence of SAT and Graves', Hoang et al report enhanced genetic susceptibility in their patient to both SAT and Graves' disease based on the HLA constitution [1].

In conclusion, here we report an unusual presentation of SAT and Graves' disease in the same patient at the same time that was accurately identified by a meticulous physical examination. Hence, it is important to remember that though rare, Graves' can present with painful goiter due to concurrent thyroiditis, or rather can be unmasked by the inflammation.

\section{Conflict of Interest}

No conflict of interest exists.

\section{Grant Support}

None.

\section{References}

1. Hoang TD, Mai VQ, Clyde PW, Shakir MK. Simultaneous occurrence of subacute thyroiditis and Graves' disease. Thyroid. 2011;21(12):1397-1400.

2. Chao CS, Lin SY, Sheu WH. Graves' disease presented as painful goiter. Horm Res. 2002;57(1-2):53-56.

3. Bindra A, Braunstein GD. Thyroiditis. Am Fam Physician. 2006;73(10):1769-1776.

4. Espinoza PG, Guendelman CL, Quevedo Limon LN, Fernandez RJ. A comparison between two imaging techniques for the diagnosis of subacute thyroiditis (de Quervain thyroiditis): brief communication. Clin Nucl Med. 2010;35(11):862-864.

5. Fujii S, Miwa U, Seta T, Ohoka T, Mizukami Y. Subacute thyroiditis with highly positive thyrotropin receptor antibodies and high thyroidal radioactive iodine uptake. Intern Med. 2003;42(8):704-709.

6. Nakano Y, Kurihara H, Sasaki J. Graves' disease following subacute thyroiditis. Tohoku J Exp Med. 2011;225(4):301-309.

7. Neufeld DS, Platzer M, Davies TF. Reovirus induction of MHC class II antigen in rat thyroid cells. Endocrinology. 1989;124(1):543-545.

8. Mousa U, Cuneyd A, Alptekin G. Should neck pain in a patient with Hashimoto's thyroiditis be underestimated? A case and review of the literature. Indian J Endocrinol Metab. 2012;16(3):444-446.

9. Bottazzo GF, Pujol-Borrell R, Hanafusa T, Feldmann M. Role of aberrant HLA-DR expression and antigen presentation in induction of endocrine autoimmunity. Lancet. 1983;2(8359):1115-1119. 